\title{
Dysgerminoma developing from an ectopic ovary in a patient with WAGR syndrome: A case report
}

\author{
RIE MIURA ${ }^{1}$, YOSHIHITO YOKOYAMA ${ }^{1}$, TATSUHIKO SHIGETO ${ }^{1}$, \\ MASAYUKI FUTAGAMI ${ }^{1}$, HIDEKI MIZUNUMA ${ }^{1}$, AKIRA KUROSE ${ }^{2}$, KAZUSHI TSURUGA $^{3}$, \\ SHINYA SASAKI ${ }^{3}$, KIMINORI TERUI $^{3}$ and ETSURO ITO ${ }^{3}$ \\ Departments of ${ }^{1}$ Obstetrics and Gynecology, ${ }^{2}$ Anatomic Pathology and ${ }^{3}$ Pediatrics, \\ Hirosaki University Graduate School of Medicine, Hirosaki, Aomori 036-8562, Japan
}

Received May 18, 2016; Accepted July 4, 2016

DOI: $10.3892 / \operatorname{mco} .2016 .1004$

\begin{abstract}
WAGR syndrome is caused by an 11p13 deletion and includes Wilms' tumor, aniridia, genitourinary anomalies and mental retardation. We encountered a case of a dysgerminoma originating in an ectopic ovary in a woman with WAGR syndrome. Our patient was a 24-year-old nulliparous woman who was diagnosed with WAGR syndrome. The patient had undergone left nephrectomy for a Wilms' tumor and postoperative chemotherapy at the age of 7 months. She also had a history of glaucoma surgery in both eyes, and was followed up at the Department of Pediatrics for diabetes mellitus, hypertension, liver dysfunction and hyperuricemia. The patient was investigated for oliguria and had elevated levels of blood urea nitrogen (45 mg/dl) and creatinine $(5.4 \mathrm{mg} / \mathrm{dl})$; she was admitted to the hospital with acute renal failure and a computed tomography scan revealed a pelvic tumor with a long axis of $10 \mathrm{~cm}$ that was obstructing the right ureter. Following insertion of a ureteral stent, the tumor was removed. The tumor had developed in the retroperitoneal space independent of the ovaries. The right adnexa were normal. The tumor was histopathologically diagnosed as dysgerminoma. Follicles were found in part of the tumor; it was thus hypothesized that the tumor developed from an ectopic ovary. The patient was administered etoposide after surgery, and has been recurrence-free for 4 years since treatment.
\end{abstract}

\section{Introduction}

A congenital anomaly syndrome including Wilms' tumor, aniridia, genitourinary anomalies and mental retardation (WAGR syndrome) is a rare, sporadic genetic disorder charac-

Correspondence to: Dr Yoshihito Yokoyama, Department of Obstetrics and Gynecology, Hirosaki University Graduate School of Medicine, 5 Zaifu-cho, Hirosaki, Aomori 036-8562, Japan

E-mail: yokoyama@hirosaki-u.ac.jp

Key words: WAGR syndrome, ectopic ovary, dysgerminoma, surgery, etoposide terized by a de novo deletion in the distal band of chromosome 11 p13 (1). WAGR syndrome is often diagnosed when aniridia is clinically evident and a chromosomal analysis is performed. The precise incidence of WAGR syndrome remains unclear. The incidence of Wilms' tumor in Caucasians is $1 / 10,000$, and a previous study reported that $0.75 \%$ of patients with Wilms' tumor have WAGR syndrome (2); thus, WAGR syndrome is an extremely rare condition. We encountered a case where a pelvic tumor in a female patient with WAGR syndrome was diagnosed as dysgerminoma. The tumor is considered to have developed from an ectopic ovary, which is defined as ovarian tissue found outside a normal ovary. An ectopic ovary is extremely rare, with an incidence of 1/93,000 (3). In addition, reports of an ovarian tumor forming from an ectopic ovary are also rare (4-6).

\section{Case report}

The present case involved a 24-year-old nulliparous woman with a height of $159 \mathrm{~cm}$, weight of $89 \mathrm{~kg}$ and BMI of $35.2 \mathrm{~kg} / \mathrm{m}^{2}$. Aniridia at birth led to the diagnosis of WAGR syndrome. At the age of 7 months, left nephrectomy and postoperative chemotherapy with vincristine and actinomycin D were performed to treat Wilms' tumor. At the age of 7 years, glaucoma surgery was performed. Since the age of 20 years, the patient has been receiving oral medication at the Department of Pediatrics of our hospital to treat diabetes mellitus, hypertension and hyperuricemia. The family history was unremarkable. The patient was investigated at the Department of Pediatrics for oliguria, and her blood pressure was 220/130 mmHg. Blood collected at the time was tested, revealing elevated levels of blood urea nitrogen (BUN; $45 \mathrm{mg} / \mathrm{dl}$ ) and creatinine $(\mathrm{Cr} ; 5.4 \mathrm{mg} / \mathrm{dl})$. The patient was admitted to the hospital with renal hypertension and acute renal failure.

Blood test results upon admission revealed a white blood cell count of $6,100 / \mu 1$, hemoglobin $(\mathrm{Hb})$ level $10.7 \mathrm{~g} / \mathrm{dl}$, platelet count $279,000 / \mu 1$, aspartate aminotransferase level $21 \mathrm{U} / 1$, alanine aminotransferase level $34 \mathrm{U} / 1$, lactate dehydrogenase $162 \mathrm{U} / 1$, total cholesterol $214 \mathrm{mg} / \mathrm{dl}$, triglycerides $298 \mathrm{mg} / \mathrm{dl}$, BUN $45 \mathrm{mg} / \mathrm{dl}$, Cr $5.4 \mathrm{mg} / \mathrm{dl}$, uric acid $7.3 \mathrm{mg} / \mathrm{dl}$, Na 144 $\mathrm{mEq} / 1, \mathrm{~K} 4.1 \mathrm{mEq} / 1, \mathrm{Cl} 104 \mathrm{mEq} / 1, \mathrm{Ca} 9.1 \mathrm{mEq} / 1$, blood glucose $101 \mathrm{mg} / \mathrm{dl}, \mathrm{HbA} 1 \mathrm{c} 5.6 \%$ and C-reactive protein $2.52 \mathrm{mg} / \mathrm{dl}$. A 

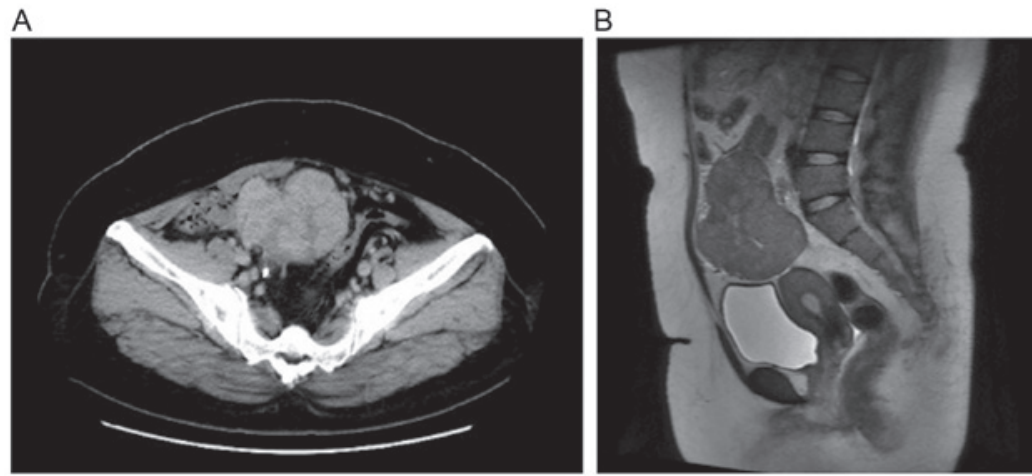

Figure 1. (A) A computed tomography scan revealed a solid mass with mild contrast enhancement that was heterogeneous. (B) A magnetic resonance imaging scan revealed a mixture of mildly hypointense areas with some hyperintensity on T2-weighted images.

A

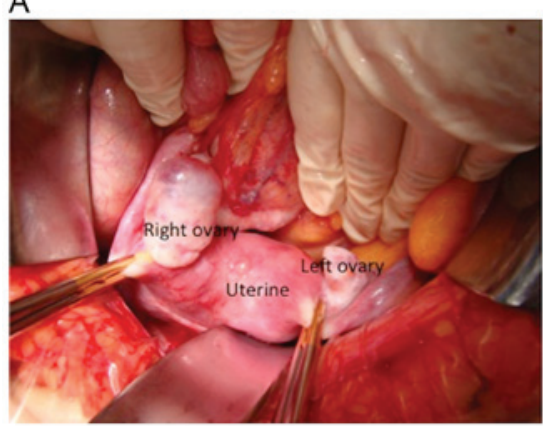

B

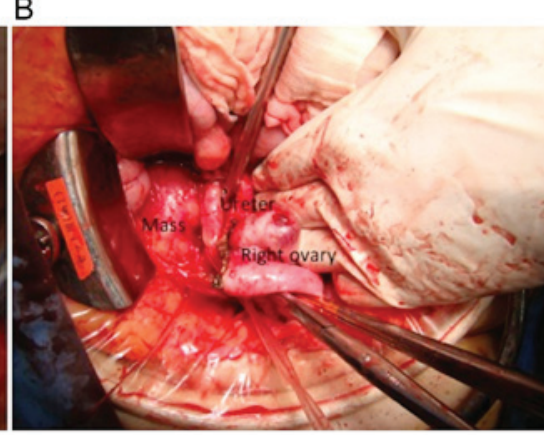

Figure 2. Intraoperatively (A) the left and right ovaries were normal; (B) the tumor was located in the retroperitoneal space behind the right ovary.

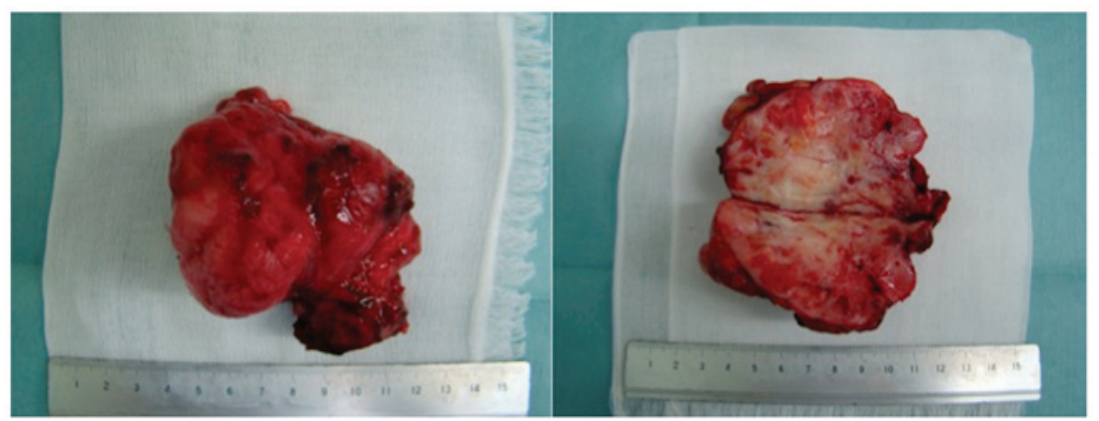

Figure 3. Resected specimen. The resected mass was solid and appeared whitish on cross sections.

CT scan revealed a solid mass with a long axis of $\sim 80 \mathrm{~mm}$ in the pelvis. There were signs of hydronephrosis in the right kidney due to ureteral compression by the mass, which was hypothesized to have caused postrenal failure. Placement of a ureteral stent in the right ureter alleviated hydronephrosis; the urinary output and blood pressure were normal. BUN and $\mathrm{Cr}$ returned to their previous levels and contrast-enhanced CT was performed. The scan revealed a solid mass with mild contrast enhancement that was heterogeneous (Fig. 1A). A tumor was hypothesized to have originated from the adnexa, so the patient was referred to the Department of Obstetrics and Gynecology. A magnetic resonance imaging scan revealed hypointensity on T1-weighted images and a mixture of mildly hypointense areas with some hyperintensity on T2-weighted images. A solid tumor without a cystic component was identified; thus, recurrence of Wilms' tumor, sex cord-stromal tumor and leiomyosarcoma were considered in the differential diagnosis (Fig. 1B). The levels of tumor markers were as follows: Carbohydrate antigen (CA) $12510 \mathrm{IU} / \mathrm{ml}$, CA19-9 $4 \mathrm{U} / \mathrm{ml}$, carcinoembryonic antigen $0.6 \mathrm{mg} / \mathrm{ml}$, squamous cell carcinoma antigen $1.2 \mathrm{ng} / \mathrm{ml}$, neuron-specific enolase $31.8 \mathrm{ng} / \mathrm{ml}$, human chorionic gonadotropin $<1.0 \mathrm{mIU} / \mathrm{ml}$ and $\alpha$-fetoprotein $2.0 \mathrm{ng} / \mathrm{ml}$.

The surgical plan was to treat a pelvic tumor originating from the right adnexa. The left and right ovaries were normal (Fig. 2A); the tumor was located in the retroperitoneal space behind the right ovary (Fig. 2B), and was not connected to the ovary. The tumor was in contact with the right ureter, but ureteral involvement was not observed. The greater omentum and retroperitoneal space were dissected, the right adnexa was spared and only the tumor was removed. The resected specimen was a solid mass, which appeared whitish on cross sections (Fig. 3). 


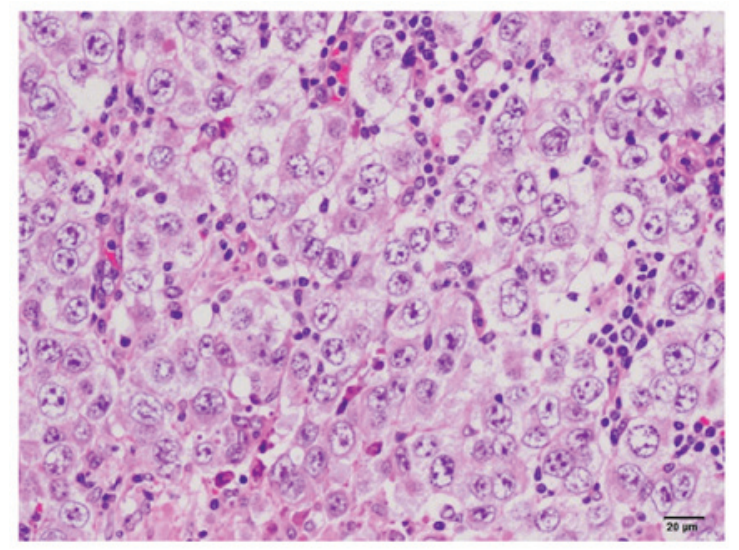

Figure 4. On histological examination, lymph node invasion from semicircular tumor cells with large semicircular nuclei. Large tumor cells and small mature lymphocytes were observed.

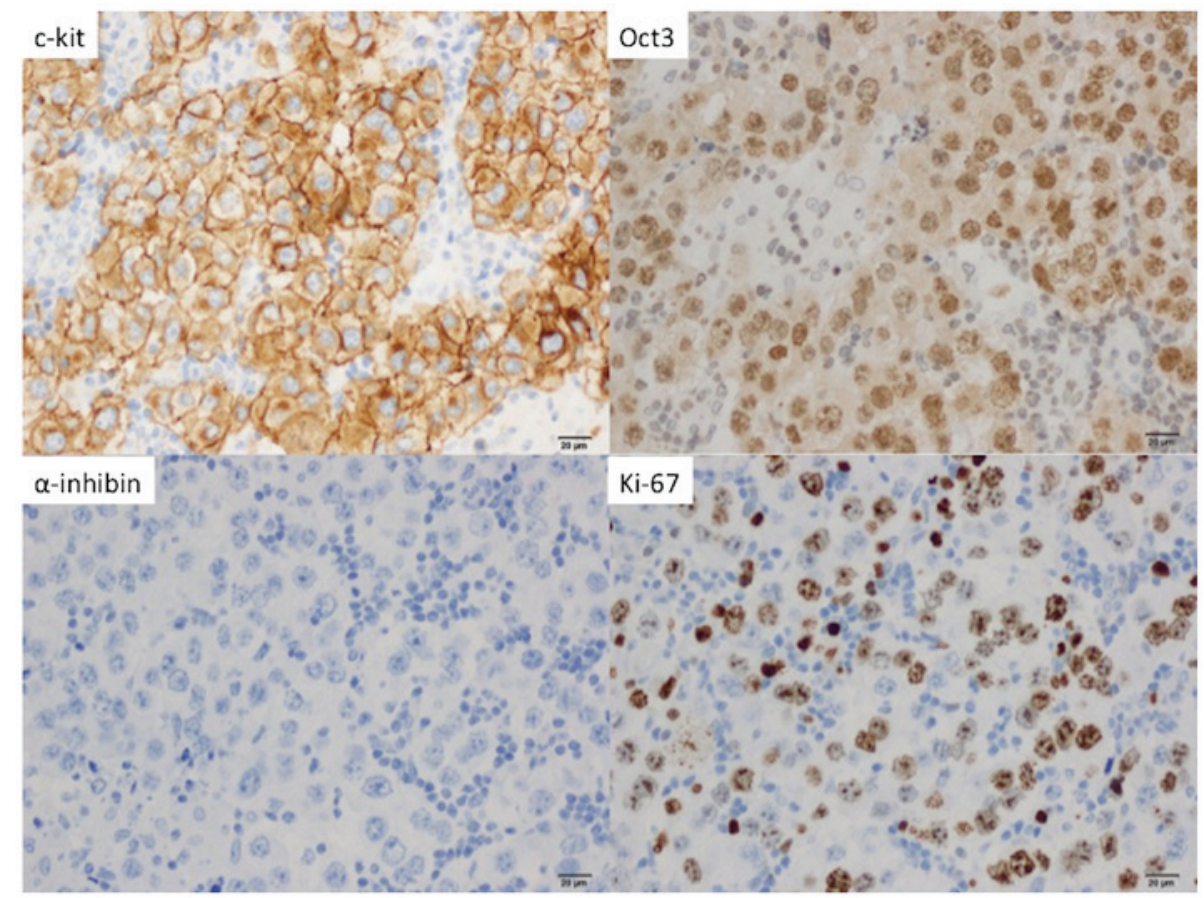

Figure 5. On immunohistochemical staining, the tumor cells stained positive for C-kit and Oct3, but negative for $\alpha$-inhibin. Furthermore, $60 \%$ of the tumor cells stained positive for $\mathrm{Ki}-67$.

On histopathological examination, lymph node invasion was observed by semicircular tumor cells with large semicircular nuclei, and a 'two-cell pattern' (i.e., large tumor cells and small mature lymphocytes) was evident. The nucleus of the tumor cells contained 1-3 nucleoli (Fig. 4). On immunostaining, the tumor cells stained positive for C-kit and Oct3, but negative for Wilms' tumor- 1 and $\alpha$-inhibin; $60 \%$ of the tumor cells stained positive for Ki-67 (Fig. 5). The final diagnosis was dysgerminoma. In addition, normal follicles were found in the tumor tissue (Fig. 6); thus, the dysgerminoma was hypothesized to have developed from an ectopic ovary.

The patient's postoperative course was uneventful. There was no solitary kidney, multiple complications, or residual tumor, so she received 6 cycles of etoposide as postoperative treatment and has remained recurrence-free in the 3 years since the surgery.

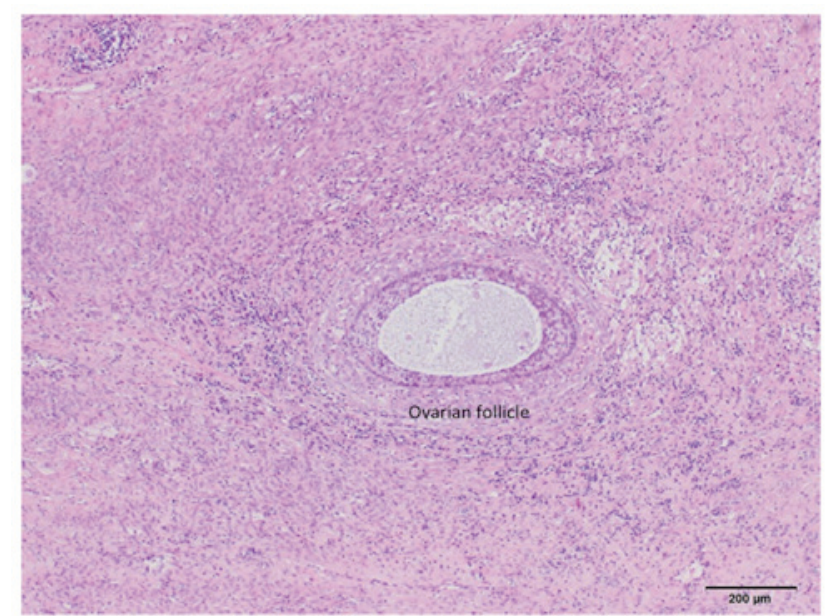

Figure 6. Normal ovarian follicle identified in the tumor. 
The patient and her family provided their consent regarding the publication of the case details.

\section{Discussion}

Ectopic ovary describes the presence of ovarian tissue outside of a normal ovary. An ectopic ovary is extremely rare, with an incidence of 1/93,000 (3). Depending on their location, ectopic ovaries may be classified as 'accessory', which are adjacent or connected to a normal ovary, or 'supernumerary', which indicates a separate location. In 1959, Wharton et al reported 2 cases of supernumerary ovaries and 1 case of an accessory ovary (7). Since that time, $<40$ cases of supernumerary ovaries alone have been reported (8), with several reported cases of both supernumerary and accessory ovaries. Lachman et al contended that the principal causes of these conditions were i) ovarian tissue that was detached during pelvic surgery and implanted elsewhere, ii) ovarian tissue that was detached due to pelvic inflammatory disease and implanted elsewhere, or iii) abnormally located ovarian tissue due to an embryological anomaly (9). The rationale for this distinction is as follows: Of the reported cases of an ectopic ovary due to ovarian tissue that was detached during pelvic surgery or pelvic inflammatory disease and implanted elsewhere, $~ 50 \%$ involved salpingitis or a history of pelvic surgery or intra-abdominal surgery. The same literature described cases of an ectopic ovary due to developmental anomalies at the fetal stage. Primordial germ cells migrate from the posterior wall of the yolk sac into the hindgut epithelium and then to the urogenital ridge, where they differentiate into ovarian tissue. When an anomaly occurs during this process of cell migration, a congenital ectopic ovary is formed. Ectopic ovaries may be found at varying sites, including the perimetrium, intestinal tract, mesentery, retroperitoneal space and greater omentum (8). In addition, diagnosing an ectopic ovary prior to surgery is usually difficult. There are case reports where an ectopic ovary was suspected prior to surgery, when follicle stimulation with clomiphene citrate resulted in follicle development within a tumor (10). In reported cases of an ectopic ovary where a tumor has formed, that tumor is most often a mature teratoma, followed by serous or mucinous adenoma (8). In some cases of an ectopic ovary, a Brenner tumor (4), Wilms' tumor (5), or epithelial ovarian cancer (6) have been reported. To the best of our knowledge, there are currently no case reports of a dysgerminoma developing in an ectopic ovary, as in the present case.

It was previously reported that genitourinary or genetic anomalies are identified in $\sim 11-36 \%$ of the cases of an ectopic ovary $(5,9)$. In the aforementioned case of epithelial ovarian cancer, the patient had Mayer-Rokitansky-Kuster-Hauser syndrome. There are also case reports of an ectopic ovary and a unicorn uterus $(10,11)$. In the aforementioned case of a Wilms' tumor, the patient had both congenital renal agenesis and an ectopic ovary. The patient in our case was diagnosed with WAGR syndrome and had a history of abdominal surgery for Wilms' tumor. The possibility that the surgery was responsible for separating ovarian tissue from an ovary is unlikely.
We hypothesize that the ectopic ovary developed as part of the WAGR syndrome.

The ultimate diagnosis of an ectopic ovary is based on the presence of ovarian cortex, follicles, granulosa cells, or theca cells (i.e., tissue specific to the ovaries) in a resected tumor. In the present case, an initial histopathological examination led to the diagnosis of dysgerminoma, although its primary origin was not known. A more detailed examination revealed the presence of follicles in the tumor. If a tumor is large, the possibility that that tumor contains ovarian tissue may be overlooked. In cases of an abdominal mass of unknown origin, the possibility of a tumor developing from an ectopic ovary must be taken into consideration, and a detailed histological examination must be performed.

In conclusion, we herein present an extremely rare case of dysgerminoma developing in an ectopic ovary in a patient with WAGR syndrome. Identifying the presence of an ectopic ovary prior to surgery is difficult, but there are reports of a malignancy developing in patients with that condition. If this condition is suspected, or a retroperitoneal tumor of unknown origin is found in the abdomen, the possibility of an ectopic ovary must be kept in mind, and a detailed histological examination must be performed.

\section{Acknowledgements}

The present study was supported by a Grant-in-Aid for Cancer Research from the Ministry of Education, Culture, Sports, Science and Technology (Tokyo, Japan) (grant no. 20591935 to Dr Y. Yokoyama).

\section{References}

1. Riccardi VM, Sujansky E, Smith AC and Francke U: Chromosomal imbalance in the Aniridia-Wilms' tumor association: 11p interstitial deletion. Pediatrics 61: 604-610, 1978.

2. Yi T, Weng J, Siwko S, Luo J, Li D and Liu M: LGR4/GPR48 inactivation leads to aniridia-genitourinary anomalies-mental retardation syndrome defects. J Biol Chem 289: 8767-8780, 2014.

3. Watkins BP and Kothari SN: True ectopic ovary: A case and review. Arch Gynecol Obstet 269: 145-146, 2004.

4. Heller DS, Harpaz N and Breakstone B: Neoplasm arising in ectopic ovaries: A case of brenner tumor in an accessory ovary. Int J of Gynecol Pathol 9: 185-189, 1990.

5. Kini H, Baliga PB and Pai KG: Supernumerary ovary associated with Wilms' tumor. Pediatr Surg Int 13: 67-68, 1998.

6. Bae HS, Ryu MJ, Kim IS, Kim SH and Song JY: Cancer of the supernumerary ovary in Mayer-Rokitansky-Küster-Hauser Syndrome: A case report. Oncol Lett 5: 598-600, 2013.

7. Wharton LR: Two cases of supernumerary ovary and one of accessory ovary with an analysis of previously reported cases. Am J Obstet Gynacol 78: 1101-1109, 1959.

8. El-Gohary Y, Pagkratis S, Lee T and Scriven RJ: Supernumerary ovary presenting as a paraduodenal duplication cyst. J Pediatr Surg Case Rep 3: 316-319, 2015.

9. Lachman MF and Berman MM: The ectopic ovary. A case report and review of the literature. Arch Pathol Lab Med 115: 233-235, 1991.

10. Uyar I, Gulhan I, Sipahi M, Hanhan HM and Ozeren M: Ectopic ovary confirmed by ovarian stimulation in a case of unicornuate uterus. Fertil Steril 96: e122-e124, 2011.

11. Ombelet W, Verswijvel G and de Jonge E: Ectopic ovary and unicornuate uterus. N Engl J Med 348: 667-668, 2003. 